\title{
A MAGNETO-OPTICAL MICROSCOPE FOR QUANTITATIVE MAPPING OF THE STRAY FIELDS FROM MAGNETIC MICROSTRUCTURES
}

\author{
W.C. Patterson, E.E. Shorman, N. Garraud, and D.P. Arnold \\ Interdisciplinary Microsystems Group, University of Florida, Gainesville, FL, USA
}

\begin{abstract}
A method is presented to quantitatively map the stray fields of microscale magnetic structures, with field resolution down to approximately $50 \mu \mathrm{T}$ and spatial resolution down to $4 \mu \mathrm{m}$. This is accomplished using a magneto-optical indicator film (MOIF), which yields an optical image of the magnetic field when observed with an upright reflective polarizing light microscope. The integrated system including the equipment, image analysis software, and experimental methods are described to extract quantitative magnetic values from optical microscopy images. MOIFs with three different magnetic field ranges have been calibrated, and the entire system is validated by measurement of the field patterns from known samples.
\end{abstract}

\section{INTRODUCTION}

Magnetic fields exist at an extremely broad range of scale, ranging in strength from pico-Tesla to 100's of Tesla. Furthermore, practical engineering systems have spatial field variations ranging in size from nanometers to 10 's of meters. Due to the large ranges in both field intensity and spatial resolution, many different methods exist for measuring and mapping magnetic fields. For example, methods such as superconducting quantum interference device (SQUID) are capable of measuring extremely small fields in the pico-Tesla range, while Hall effect sensors can measure fields up to the 100's of Tesla.

Our interests lie in magnetic microsystems, e.g. microscale electromagnets or permanent micromagnets integrated on chip, where the magnet dimensions may range from $1 \mu \mathrm{m}$ to 100 's of $\mu \mathrm{m}$. To map the stray fields from such structures, we desire a field measurement tool capable of measuring fields ranging from $\mu \mathrm{T}$ to $\mathrm{T}$, while mapping over distances of millimeters with a spatial resolution of $\leq 1 \mu \mathrm{m}$. Table 1 outlines three forms of field mapping [1 - 3] that fit the desired range for magnetic MEMS work, each with their own pros and cons. The first two, magnetic force microscopy (MFM) and scanning Hall probe microscopy (SHPM), are raster methods of field mapping. Due to this rastering, the scans of MEMS scale structures take an immense amount of time, and the required equipment is fickle and often quite expensive. In contrast, magneto-optical imaging (MOI) is an imaging technique that affords the benefit of speed, along with low cost and ease of operation, which outweigh its shortcomings in sensitivity and spatial resolution.

Table 1: Magnetic measurement technique comparison. Red $=$ Bad, Yellow $=$ OK, Green $=$ Good

\begin{tabular}{|l|c|c|c|}
\hline & MFM & SHPM & MOI \\
\hline Measurement & Force & $\mathrm{B}_{\mathrm{z}}$ & $\beta \propto \mathrm{B}_{\mathrm{z}}$ \\
\hline Sensitivity & $1 \mu \mathrm{m} / \mathrm{N}$ & $0.1-10 \mu \mathrm{T}$ & $>50 \mu \mathrm{T}$ \\
\hline Spatial res. & $20 \mathrm{~nm}$ & $0.5 \mu \mathrm{m}$ & $>1 \mu \mathrm{m}$ \\
\hline Scan size & $300 \mu \mathrm{m}$ & 10 's mm & 100 's mm \\
\hline Field range & Broad & $\pm 16 \mathrm{~T}$ & $\pm 500 \mathrm{mT}$ \\
\hline Scan speed & $<100$ points/s & $<10$ points/s & $<30 \mathrm{frame} / \mathrm{s}$ \\
\hline
\end{tabular}

This paper presents a tool called a "magneto-optical microscope" for quantitative imaging and measurement of stray magnetic fields produced from micromagnetic structures. The capability for large-area, high-spatial-resolution measurement of
milli-Tesla to Tesla-level magnetic fields fills a critical metrology gap for magnetic microsystems, not served by MFM or SHPM [1]. Benefits of our system are: high magnetic resolution (ranging $\pm 50 \mu \mathrm{T}$ to $\pm 1 \mathrm{mT}$ ), fast characterization (few seconds) over a large spatial area $\left(\sim \mathrm{cm}^{2}\right)$, high spatial resolution (ranging $4.2-20.1 \mu \mathrm{m}$ ), non-destructive, non-invasive, non-contact, and relatively inexpensive total hardware cost $(\sim 50 \mathrm{k})$. The system was designed to require very little custom hardware and to be fairly flexible to varying measurement requirements, as commonly found in a research lab. Herein we report the system design, operation, calibration, and validation.

\section{SYSTEM OPERATION \& DESIGN Magneto-Optical Imaging Film}

At the heart of the system functionality is the MOIF, which leverages the Faraday Effect for visualization and measurement of magnetic fields. As illustrated in Figure 1, the Faraday Effect is an interaction between light and a magnetic field in a medium, which causes a rotation of the plane of polarization in proportion to the magnetic field [4].

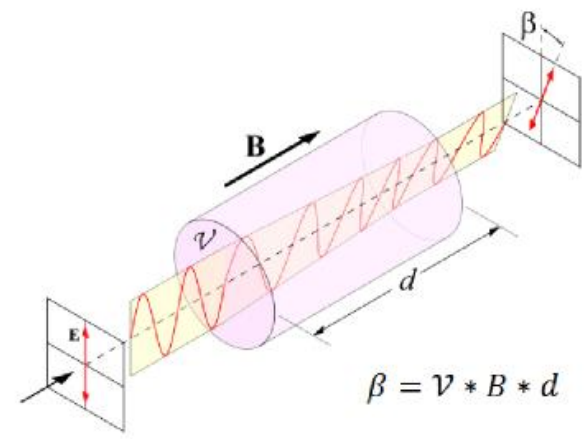

Figure 1: Diagram illustration of the Faraday effect. Diagram represents an example of light transmission completely through a material. On our MOIF one side is reflective, so polarization rotation is further exaggerated as light reflects back through the material again. ("Faraday-effect" by Bob Mellish / CC BY).

As shown in Figure 2 the MOIF is a multi layer sensor that consists of: an optically transparent substrate layer that provides mechanical support and the correct crystal structure for film growth, a magneto-optically active layer (MOL) that has a high Verdet constant $v$ resulting in a strong Faraday Effect [5], a mirror coating to reflect the light back through the MOL again, and a protective layer made of a high hardness material to protect the mirror and MOL.

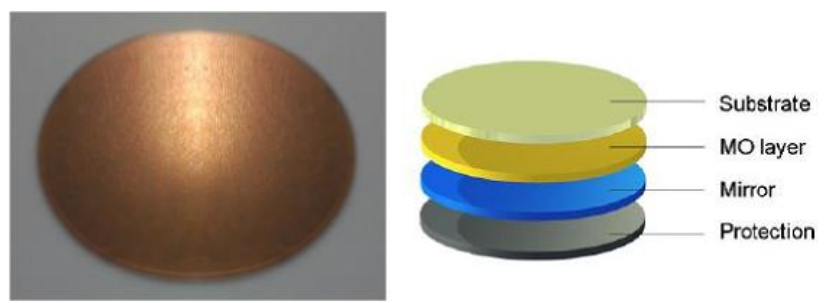

Figure 2: (Left) Commercial MOIF from Matesy, GMBH. (Right) Exploded view of MOIF layers. Hilton Head Island, South Carolina, June 8-12, 2014 
The MOL material used in this system is a bismuth substituted yttrium iron garnet (Bi-YIG) grown on a gadolinium gallium garnet (GGG) substrate. The reflective coating is aluminum with a sapphire protective layer deposited on top. The films are purchased from Matesy GMBH, and different saturation/sensitivity films are available. The current system has three MOIFs available with 5, 40, and $250 \mathrm{mT}$ saturation ranges.

\section{Magneto-Optical Microscope System}

A block diagram of the magneto-optical microscope is shown in Figure 3. The system is designed around an upright metallurgical microscope for the sake of cost and ease of use. In operation, the MOIF is positioned in close proximity to the magnetic sample to be measured. White light from the standard microscope bulb is linearly polarized and then passed down through the microscope objective. The light then passes through the transparent substrate, translucent magneto-optic layer, and reflects off the mirror coating on the bottom side of the MOIF (see Figure 2). During this transit, the polarization is rotated in proportion to the perpendicular (out-of-plane) magnetic field strength. The rotated light passes back up through the objective and is filtered by the analyzer, which is a second linear polarizer. After the analyzer, the light has an intensity distribution that is proportional to the magnitude of the magnetic field sensed by the MOIF. This intensity image is then captured by the grayscale camera and creates a visual representation of the stray magnetic fields.

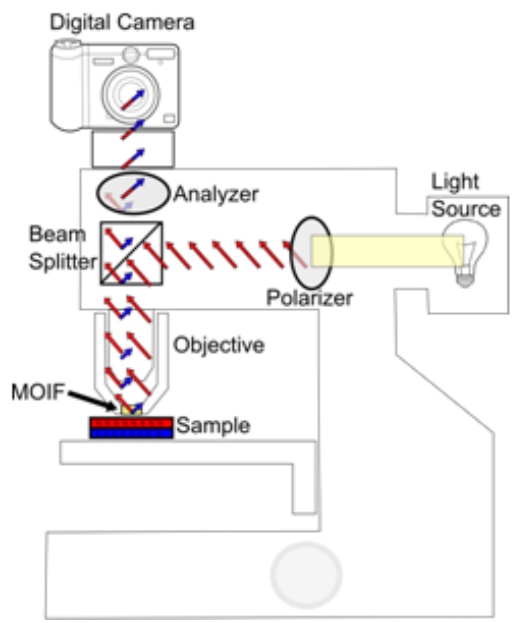

Figure 3: Block diagram of quantitative MOI system.

\section{Magneto-optical Microscope Components}

The magneto-optical microscope is shown in Figure 4 and consists of four main components: a reflective polarizing light microscope (Olympus BX51), a grayscale digital camera (Thorlabs DCC1545M), a milled objective sleeve holding the MOIF, and custom digital image processing software.

A grayscale digital camera was chosen due to the difficulties that were encountered in preliminary experiments using a color camera. Under white light illumination, the MOIF exhibits a shift in color in response to a magnetic field. This color shift and the nonlinearities in the pixel color filters used in a color camera, made it difficult to obtain pixel intensity values without saturating some colored pixels, or leaving others without signal completely. The grayscale camera that is used in the system has a fairly low-quality CMOS imaging sensor. Low-noise data can still be obtained by averaging multiple images. For higher resolution images, a larger, scientific quality, grayscale CCD camera could be acquired.

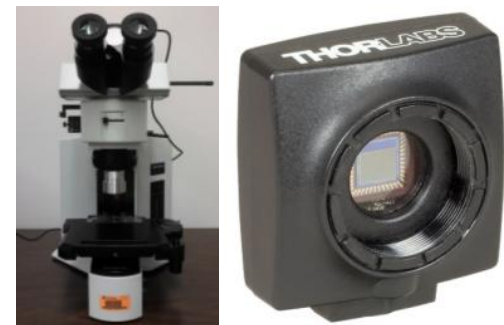

Figure 4: (Left) BX51, upright reflective polarizing light microscope, with MOIF sleeve slid on to objective. (Right) Thorlabs DCC1545M grayscale digital camera.

Previously, MOI processing required that the MOIF be placed directly onto the sample, and then the microscope be focused onto the MOIF to obtain the image. We've found the following drawbacks with this approach:

- It requires that the MOIF be placed carefully on the samples area of interest. Often the desired sample does not have any visible markers apparent to the naked eye making the placement challenging.

- There is a limited measurement area before the MOIF needs to be repositioned.

- Every image is taken on a different area on the MOIF. The MOIF is not necessarily uniform so the calibration becomes difficult.

- The light path can be affected by outside sources, such as the rooms' ambient lighting.

- The MOIF is limited to only measuring at the samples surface.

- The focus is done manually per sample and may cause variability in measurements.

To solve these problems, a sleeve was machined to mount the MOIF onto the microscope objective, as shown in Figure 5. The MOIF is positioned at the focal plane via the adjustable nose piece on the sleeve. The benefits of the sleeve are that: it allows the MOIF to remain at the focal point of the microscope as samples are changed or moved; it prevents the MOIF from rotating or translating; it eliminates all outside light sources; and the spring loaded nose piece can assure repeatable sample contact pressure.

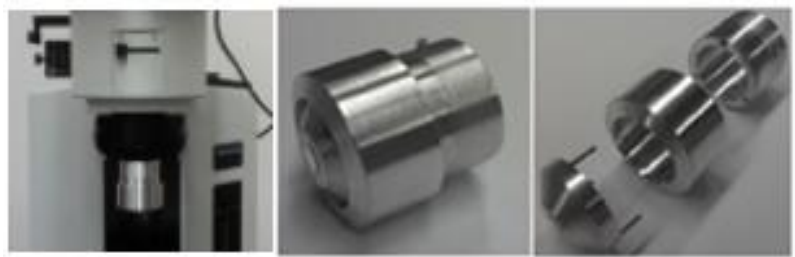

Figure 5: (Left) Custom optic sleeve installed on objective. (Middle) Assembled optic sleeve. (Right) Exploded view of sleeve.

\section{Image Analysis Software}

To achieve quantitative data from the images, a reliable correlation must be made between the grayscale intensity values from the camera and the B-field values acting on the MOIF. Unfortunately, making this correlation is hindered by variations in total light intensity, camera exposure time, light path depolarization, and optical non-linearities in the optical path, and electronic non-linearities associated with the camera [5].

All of the previously mentioned signal corruptions could be considered "common mode" noise and could be rejected by differential imaging. Indeed, there is an absolute correlation 
between the differences of two identical grayscale images with inverse analyzer crossings (e.g. $\pm 15^{\circ}$ ). With this in mind, a novel method is introduced using three images with symmetric analyzer settings to create a quantitative image. The three images are taken with different analyzer settings, $-15^{\circ}, 0^{\circ}$, and $+15^{\circ}$. Then the individual pixels of the grayscale intensity image are entered into the following equation:

$$
\Delta=\frac{I_{-15^{\circ}}-I_{+15^{\circ}}}{I_{-15^{\circ}}+I_{+15^{\circ}}-2 I_{0^{\circ}}}
$$

The resulting delta values create a "normalized image." This normalized image represents only the rotation of the polarization plane, rejecting all the "common mode" error signals described above. Figure 6 shows an example calibration curve/matrix for the MOIF at known magnetic fields supplied by a calibrated electromagnet. When making measurements, the pixel intensities are mapped to their corresponding B-field values to form the magnetic image.

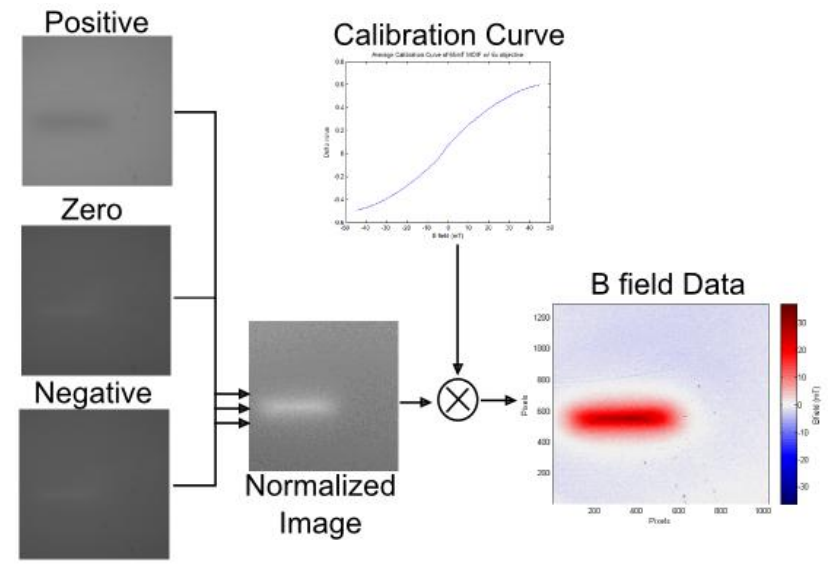

Figure 6: Steps in the quantitative MOI process.

\section{PROCEDURE}

\section{Calibration Procedure}

The calibration process creates a curve relating the delta value to B-field for every pixel in the image. A representative calibration curve for the entire MOIF is created by averaging all of the pixels calibration curves. An example can be seen in Figure 7.

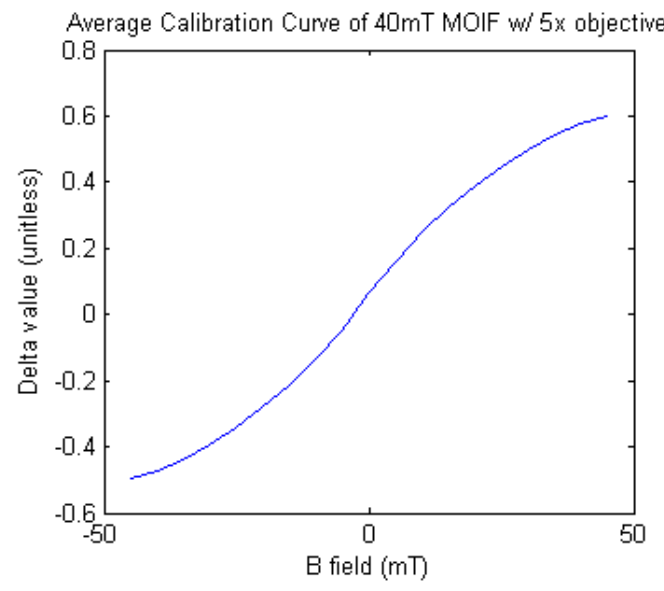

Figure 7: Calibration curve of $40 \mathrm{mT}$ MOIF with $5 \times$ objective. $B$ field is swept from -40 to $+40 \mathrm{mT}$, resulting in a normalized value proportional to polarization rotation.
To create a calibration curve/matrix, the MOIF is subjected to a known uniform field, a delta image is obtained, the field is increased, and the process is repeated across the desired field range until the entire calibration curve/matrix is obtained. To achieve a uniform field, an air core solenoid was designed and wound to fit on the microscope stage around the MOIF sleeve. The field strength and spatial uniformity of the solenoid was then calibrated using a DC current supply and an instrument grade gauss meter (Lakeshore 475 DSP Gaussmeter).

\section{Quantitative Imaging Procedure}

To measure unknown samples, three images are taken of the MOIF at three different polarizer settings: $-15^{\circ}, 0^{\circ}$, and $+15^{\circ}$. These pixel intensity values of these images are calculated via (1) in order to create a delta image. This image is then interpolated against the calibration data. The result is a quantitative map of the stray magnetic fields of the user's sample.

\section{RESULTS}

\section{Calibration}

The three different ranged MOIF's were re-calibrated five times over a two-week period to test the repeatability of the MOIF and setup procedure. A representative repeatability test is shown in Figure 8. The overall shape and peak-to-peak amplitude remain consistent. However, there is a small DC offset from calibration to calibration. It was found that the offset is caused by miniscule $\left(<1^{\circ}\right)$ changes in the absolute position of the analyzer setting. To prove this, three calibrations were performed with the analyzer settings at approximately $\left(-14^{\circ}, 1^{\circ}, 16^{\circ}\right),\left(-15^{\circ}, 0^{\circ}, 15^{\circ}\right)$, and $\left(-16^{\circ}\right.$, $\left.1^{\circ}, 14^{\circ}\right)$. This caused the curve to be offset on the y-axis with no change in curve shape or amplitude.

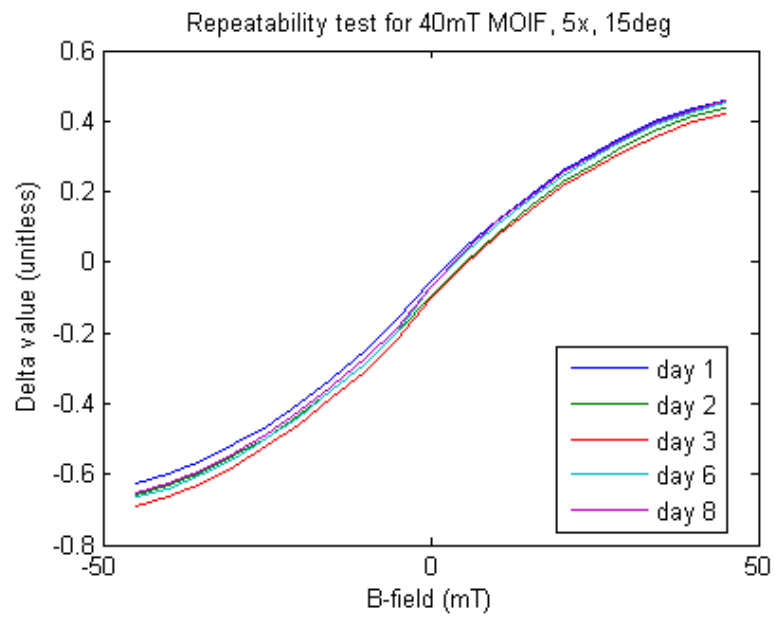

Figure 8: Example calibration repeatability curves for the $40 \mathrm{mT}$ MOIF over an 8 day period.

\section{Validation/Example Measurements}

As a proof of operation, a known sample is measured using the magneto-optical microscope. The sample chosen for this test was a commercially purchased $\mathrm{NdFeB}$ cylindrical magnet with a $1.0 \mathrm{~mm}$ height and $1.0 \mathrm{~mm}$ radius. The magnetic properties were first measured using a vibrating sample magnetometer (VSM), and from this information the stray magnetic fields were simulated using finite element analysis software (COMSOL Multiphysics). The stray fields were then experimentally measured using the $250 \mathrm{mT}$ MOIF. Multiple measurements were taken at different heights above the sample. 
Figure 9 is a comparison plot between the experimental results and the simulations. Only three heights are shown for clarity. The experimental results match the simulations fairly well. Discrepancies could be caused by slight inaccuracies in the simulation due to magnet size tolerance and a thin protective nickel coating on the outside of the magnet being unaccounted for. On the experimental side, there is some uncertainty in the height above the sample; this error would create a larger effect in measurements closer to the sample surface.

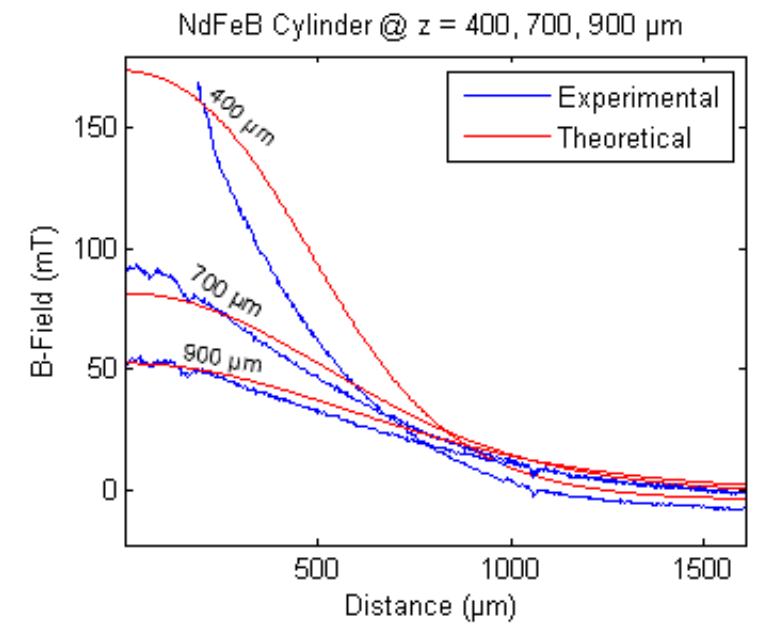

Figure 9: Experimental versus theoretical cross section measurements of a $1 \mathrm{~mm} \mathrm{NdFeB}$ cylinder magnet at 400,700,and $900 \mu \mathrm{m}$ above the surface.

An example of a more complex field is seen in Figure 10. This is a two dimensional map of the field intensity at the surface of an interdigitated magnetic micro-undulator [6]. In this sample each finger is magnetized in the opposing direction. The black strips at the center of the fingers are points where the field exceeds the maximum measurable field of the MOIF; at these points the field is not defined (saturated points are plotted black).

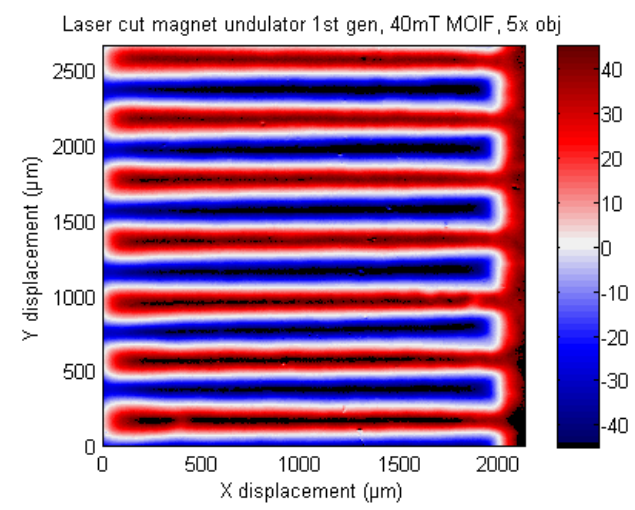

Figure 10: Quantitative image of the alternating field pattern from an interdigitated magnetic micro-undulator.

\section{System Specifications}

The measurement specifications of the system are summarized in Table 2. Magnetic resolution is calculated by taking the saturation range for a particular MOIF, and dividing it by the eight bit camera resolution. Spatial resolution is calculated as the total MOL, reflective layer, and protective layer thickness [4]. Actual spatial resolution may vary depending on flatness of the sample, and precision of testing techniques used by the operator. This is because any sample to MOIF gap will be added to the spatial resolution calculation.

Table 2: Minimum specifications for calibrated MOIFs.

\begin{tabular}{|c|c|c|c|}
\hline $\begin{array}{c}\text { MOIF } \\
\text { Type }\end{array}$ & $\begin{array}{c}\text { Magnetic } \\
\text { Range }\end{array}$ & $\begin{array}{c}\text { Magnetic } \\
\text { Resolution }\end{array}$ & $\begin{array}{c}\text { Spatial } \\
\text { Resolution }\end{array}$ \\
\hline $\mathbf{5} \mathbf{~ m T}$ & $\pm 5 \mathrm{mT}$ & $\pm 50 \mu \mathrm{T}$ & $4.2 \mu \mathrm{m}$ \\
\hline $\mathbf{4 0} \mathbf{~ m T}$ & $\pm 40 \mathrm{mT}$ & $\pm 0.5 \mathrm{mT}$ & $6.2 \mu \mathrm{m}$ \\
\hline $\mathbf{2 5 0} \mathbf{~ m T}$ & $\pm 230 \mathrm{mT}$ & $\pm 1 \mathrm{mT}$ & $20.1 \mu \mathrm{m}$ \\
\hline
\end{tabular}

\section{CONCLUSIONS}

We have developed a system and unique image processing system to quantitatively map the stray fields of microscale magnetic structures. This MOIF-based optical measurement technique allows large areas to be measure nearly instantaneously while still maintaining a useful measurement range $( \pm 5 \mathrm{mT}$ to $\pm 230 \mathrm{mT})$ and resolution $( \pm 50 \mu \mathrm{T}$ to $\pm 1 \mathrm{mT})$. The system is built using common lab equipment and materials. By using a novel differential imaging method, common mode signal errors can be easily removed. The technique was validated by comparing the data obtained from the magnetic microscope of a known sample against its theoretical model. This system provides a valuable measurement capability to aid in the ongoing development of miniaturized electromagnets, magnetic materials, and other related microsystems.

\section{ACKNOWLEDGMENTS}

This work was supported in part by Army Research Office (W911NF-09-1-0511), DARPA (N6601-11-1-4198), and Allegro Microsystems LLC.

\section{REFERENCES}

[1] S. J. Bending, "Local Magnetic Probes of Superconductors," Advances in Physics, 1999.

[2] J. H. Kindt, "Survey, Screening, Dynamics: A No-Compromise Approach to High-Speed Atomic Force Microscopy," Bruker Nano Surfaces, Santa Barbara, CA, USA, 2011.

[3] F. Laviano, et al., "An improved method for quantitative magneto-optical analysis of superconductors," Supercond. Sci. Technol., vol. 16, p. 71-79, 2003.

[4] R. W. M. Koblischkat, "Magneto-optical investigations of superconductors," Supercond. Sci. Technol., vol. 8, 1995.

[5] B. Azzerboni, et al., "Magnetic Imaging Films," in Magnetic Nanostructures in Modern Technology, Springer, 2008, p. 195224.

[6] B.A. Peterson, et al., "Laser Micro-Micromachined Permanent Magnets With Highly Spatial Alternating Magnetic Field Distribution," in PowerMEMS, Dec. 2012.

\section{CONTACT}

*William C. Patterson; wmcpatterson@ufl.edu 